\title{
Research on the Architecture of Future Library Based on Big Data
}

\author{
Ming Li , Yongcheng Luo
}

Library Yancheng Institute of Technology, Yangcheng,Jiangsu Provice, China

Library \& College of Information Science and Technology. Donghua University Shanghai, China

Im@ycit.cn, lycluo@dhu.edu.cn

Keywords: Future library, Library strategy, Architecture, Big data, Library's death.

\begin{abstract}
With the maturing of big data, big collaboration, large platform technology, the library' pattern becomes one of the hot issue in the library domain experts. This article reviews the main idea about the future library at home and abroad. Then, based on the trends of big data, big collaboration, large platform technology, the whole architecture of future library is produced, and described in detail from users reception, library's collections, services, environment, status and other aspects. Then, librarians must strengthen their information literacy and knowledge skills to meet the demands of future library. Service provided by the library will be ever-present and ubiquitous, and it will connect people and people, people and literature closely.
\end{abstract}

\section{Introduction}

With the maturing of big data technology, its applications are gradually popular in various industries. There are many applications of big data, big collaboration and large platform in technological innovation, e-commerce, market forecasting, product evaluation and other areas. With the deep application of big data in the library, traditional library has had to take the corresponding change.

Library "death theory" is accompanied by the development and reform of modern information technology. Experts in the area of the library discuss this issue have continued without interruption. In the 1970 of the 20th century, United States library scientist F.W. Lancaster first suggested the library "death theory" [1]. Subsequently, Brian T.Sullivan of Alfred University Library published the "2050 University libraries in the coroner's report” in 2011 [2]. New Fortune magazine put forward "the librarian profession after 15 years will cease to exist" in November 14, 2014 [3].

\section{Some Ideas of the Future Library}

Library is the frontier of information service, text mining, competitive intelligence, value statistics and other areas. So, there is an urgent need for library to use big data technology build the big platform to provide service for users, innovation and society.

How to deal with and reform in big data environment. Domestic and foreign scholars have conducted a lot of research and exploration. Many ideas have been proposed. For example, rebuilding library as "no book library" [4], establishing digital service paradigm which completely change the library organization culture, people in the organization and working way, established academic tradition [5], reconstructing the library's user-centered business model and re-building new library paradigm adaptable to digital environment [6], building resources, services, and technology integrates future library [7]. Wilfred Suhl-Strohmenger thinks that the center of future library is user, and future library will provide access to a wide range of physical and digital resources and services for those users who have information literacy [8]. .

\section{The Whole Model of Future Library}

Future library is a collection of big data, big analysis, big processing and big service for the physical and virtual build symbiotic service system. Based on the big data, big collaboration, large platform technology trends, I would like to propose a general architecture for future library, as shown in Figure 1. 
It collects various data by some library foundation layer facilities, such as smart building, wireless network, Internet of Things and intelligent sensing devices. And these data are massive, containing paper documents, digital data, using data, system data and so on. Future library can provide decision support services through big data technology, containing text mining, competitive intelligence, value statistics and econometric analysis. At the same time, librarians will launch embedded service timely, and provide the user with the ever-present, ubiquitous service. Library becomes the smart library in true sense. And its reception, collections, services, environment and status have changed a lot.

\section{A. Users Reception}

When the user through the unified identity authentication, the library would give him information he may need by intelligent way. The user who walks into virtual library or physical library will conduct a uniform identity authentication firstly, then the library will make data analysis according to his reading of library history, hobbies, reading habits and other information. Robots in library (or virtual robot) will provide the user with a personalized custom service APP. Besides, users can keep abreast of personal history behavior, personal preference information push, information commons (IC) utilization and reservation, the development of related disciplines at home and abroad, history of literature and information use and other information, and can also get a customized library service platform by APP.

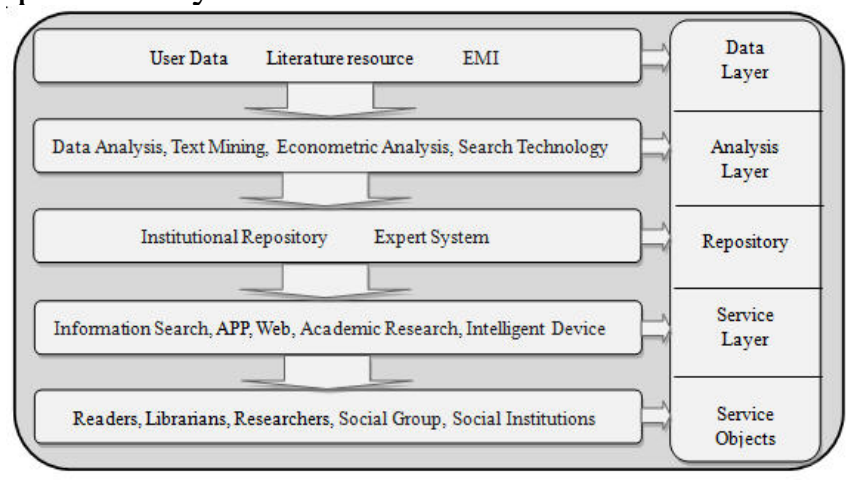

Figure 1. The whole architecture of future library.

Future library will be users' self control library, so as to form a whole "Library-User" one. The big data, big collaboration, and large platform in the background support more deep information services in library and information technology service. Literature information provided by APP will not only have the characteristic of today's MARC data, and it will also provide the clustering information by combining economic value and reading reviews and other aspects. The user enters library just like entering personal study. They can customize a personal library according their reading preferences and academic interests, and can also complete this work with the help of librarian.

\section{B. Collection}

Future library no longer needs a consistent layout bookshelf to organize books, but centralized storage. Users can get reserved resource at designated location by robot. At the same time, the kind of books and the source of resources become more widely.

Future library will no longer be a monotonic space and consistent layout library which is full of paper literature resources, and the entity collection will be gradually into the background centralized warehouse. Besides, library will establish unified electronic identity information for all storage literature, and the entity collection will no longer be confined to the general document type such as books, periodicals, newspapers, but will cover various fields including humanities, science and society, and will cover various aspects including individual designs, manuscripts and works. When the user gets entity collection literature, he only needs to complete the lending and reservation formality by APP, that via the APP' s prompt, at the specified time and the specified window to lend literature resource with the help of document storage center's robot.

Data providers will gradually withdraw from the stage of library history, and OA resources will 
reach unprecedented prosperity. Future library will no longer be a show place for data providers' search platform, but for metadata information under the guidance of big data technology. Future library only collects the entity collection of documents and electronic documents with metadata, and provides the metadata interface of electronic identity information for every visitor. Users can choose self purchase, open source code and searching platform of library to retrieval, customize, organize and analyze literature information.

The barrier of literature information sharing will no longer exist. Future library will no longer be a small but perfectly formed library, the entire collection of documents will no longer be the final goal of every library. Future library will be a definite theme library, and books, journals, magazines will no longer be the main collection of library literature information sources. All goods have the characteristic of literature will become the resource of future library. While the future library receives user, the library APP will provide all metadata access interface of library sharing agreement. Besides, users can retrieve and use all metadata of library sharing agreement by library APP, and can achieve a lot of function, like consulting and reserving literature resources.

\section{Service}

Future library pays more attention to intelligence. The original work must be completed by the librarian, now can be done by a robot or other intelligent equipments. The Figure 2 shows the service process of future library.

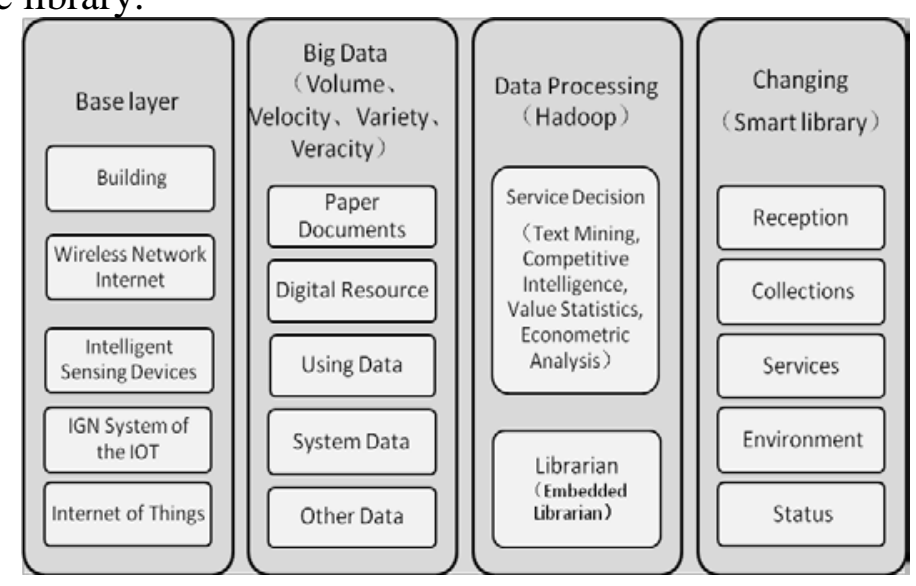

Figure 2. The service process of future library.

The staff of future library will be freed from the process and collection of a large number of complex literature resources, sorting of books and periodicals, management of access channel machine and antitheft, consulting and explanation services and so on. The future library service is divided into three aspects: application and popularity of modern information technology in the library, classification and indexing and alternative measurement of the literature information, the subject or topic cluster of literature and text mining. With the gradual application of big data, big environment, large platform technology in the future library, the position of modern information technology will be increasingly important, especially manufacture and promotion of themed or personalized APP, construction and application of the library proprietary application platform, improvement and popularity of open source applications. These will directly determine the development and status of the library. Future library will establish specialized agencies to implement application and popularity work of the modern information technology in the library, and it will support the survival and development of future library, and also carry the extension of library services in the future.

Future library consists of a number of library with distinct theme, and big collaboration will be the basic premise of the cooperative development of regional library, index of theme literature information, and all this will directly determine the level and quality of library to provide literature information. Library consortia will reserve some special fields specifically, such as alternative measurement and clustering analysis of text, on the basis of the formulation of literature information in international standards or national standards. The information service staff of future library will use the relevant field information, according to the user's needs, and generate statistical 
analysis report of literature, institutional repository or subject / thematic cluster of literature report on the basis of intelligent analysis and text mining.

The reference and consultancy service of the future library will be replaced by a more professional information push service, and most of today's reference and consultancy function will be replaced by the self-help robot in future library. The future library information push service will pay attention to the continuation and advance of some intelligent services, such as users' behavior analysis, literature value analysis, text mining and cluster analysis of literature, user knowledge management delegation, subject of competitive intelligence analysis, alternative measurement of document information and institutional repository construction, key subject science information trace, research competition of academic institutions and so on, all of that on the basis of some services, like personalized users' information consulting service, literature clustering arrangement, special topic analysis report.

\section{Environment}

Future library is not full of books, but smart learning environment. The user can receive the services they need through smart devices, and the librarian provide service through smart devices.

The future library environment will be divided by three functional modules: functional area of collection, IC, intelligent service and technical support. IC will be focal point of the library construction and core of library service. Besides, there will build a dedicated channel (containing convenient communication channels, APP communication platform, easy video communication interface and so on) among IC, functional area of collection, intelligent service and technical support area.

Today's modern information equipment in the library will no longer be a mainstay of future library, users can get perfect service and the user experience in anywhere of the library through intelligent digital equipment and library APP. Future library will not see the full of books, periodicals and newspapers, will not see the same tables and chairs and sofas, will not see the large library and reading room, also will not see the full modern information equipments. Future library will be a set of leisure, learning, research and emotion in one, and may be separated into different IC, which is independent or academic or comfortable or entertaining. What's more, according to the users' needs, they can reserve and use the IC.

\section{E. Status}

The function of future library will more completely. Librarians take more cultural and social responsibilities, and will get higher recognition.

The future library will not only be an important place for users to gain knowledge, and will also assume propaganda and popularity of the regional culture, society, legal knowledge. At the same time, it is the distribution and storage center of regional literature resources, human resources, social resources, scientific research information resources and achievements information resources. Future library will open up a dedicated area in the IC for users as the place of academic research, academic training and knowledge dissemination. Future library will no longer be just a user reading place, but will combined closely with regional economic, regional society and regional human as a whole.

The future librarian will do more than just working in an enclosed area, and serve not only to the specific audience. On the contrary, the librarian's service will be extended to economic, society, culture and other fields, and will take more social responsibilities and cultural responsibilities. Besides, they should provide the necessary literature information and value support for decisionmaking of the regional economy, regional society and regional human behavior, and undertake the collection, collation and sharing of information on work-related documents which in regional level relating to economic, society and culture, and undertake the regional library, cooperation and joint working of subject library in big environment. Future status of librarians will get higher recognition and respect in the social dimension, and will be communicator of promoting regional economic, society and regional human development. 


\section{F. Strategies for librarians}

Responsibilities of librarians will change as the library functions vary. Librarians must strengthen their information literacy and knowledge skills, only doing like this can meet the demands of future library which in the big data environment. In order to respond to and deal with the challenge of the future library, and provide better services to users, librarians should strengthen their basic knowledge and skills from the following several points. First, strengthen the sense of service, establish a "users first, service first" point of view; Second, strengthen their capacity of understand and reprocessing the information; Third, improve their foreign language skills and language expression ability; Fourth, master big data technologies, including high performance big data analysis, MPP Computing, In-Database Computing and so on; Fifth, have the relevant knowledge of network, including the establishment of LAN and network trouble shooting; Finally, strengthen resource storage and management capacity. All in all, future library is not just of advanced equipment and technology, as well as advanced librarians. In order to meet the needs of future library more better, the librarians need to keep absorbing new knowledge, mastering new technology, then they so as not to be eliminated in the future.

\section{Conclusion}

The era of big data, big collaboration and big platform has arrived, library requires the integration of modern information technology, caters to users' needs for library services and information in all aspects, becomes part of the human society. Future library integrate resources, services and technologies in the big data environment, and is different from traditional library in reception, collection, service, environment, status and so on. Besides, service provided by the library will be ever-present, ubiquitous, and it will connect people and people, people and literature closely.

\section{Acknowledgment}

This research was supported in part by the Fundamental Research Funds for the Central Universities and the joint laboratory of Donghua University and Dameng Database Corp. Second author is corresponding author.

\section{References}

[1] Lancaster F W, "Libraries and librarians in an age of electronics," Arlington: Information Resource Press,1982.

[2] Sullivan B T., “Academic library autopsy report,2050,” [2011-01-06].http://chronicle.com/ article/Academic-Library-Autopsy/125767/.

[3] New Fortune Magazine. "15 years later, the 12 kinds of occupation will disappear.Your job still available?” [2014-11-14]. http:// www.wtoutiao.com/a/723867.html.

[4] "Stanford University library going bookless," [2010-11-22]. http: //blogs.sacbee.com/mt/mtsearch.cgi?blog_id=11\&tag= bookless\%20 library\&limit=20\&IncludeBlogs=11.

[5] Carnaby P., "Connecting the knowledge networks: Reimagining academic libraries to 2020 ," [2011-09-15]. http://conference.ifla.org/ sites/default/files/files/papers/ifla77/122-carnabyen. pdf.

[6] Chu Jingli,Yang Zhigang, “Survive or Die: Critical Review of the New Statement of Libraries' Death,” Library and Information Service, 2012,56, vol.11, pp. 5-11.

[7] Zhang Haiqing, Zhangcheng, "The Crisis and Reform of Library: Thoughts Concerning the Reorganization of Harvard University Library.Journal of Library and Information Sciences in Agriculture, 2013,25,vol.6 , pp. 109-111. 
[8] Sühl-Strohmenger W., “Libraries' futures, user needs and information,” [2012-03-10]. http://www.nb.admin.ch/aktuelles/auss tellungen_und_ veranstaltungen/00726/01612/index.html?lang=en. 